\title{
Matrix metalloproteinases 2 and 9 and MMP9/NGAL complex activity in women with PCOS
}

\author{
Javad Ranjbaran, Marzieh Farimani ${ }^{1}$, Heidar Tavilani, Marzieh Ghorbani ${ }^{1}$, Jamshid Karimi, \\ Faranak Poormonsefi ${ }^{1}$ and Iraj Khodadadi \\ Department of Biochemistry, Faculty of Medicine, Hamadan University of Medical Sciences, Hamadan, Iran and \\ ${ }^{1}$ Endometrium and Endometriosis Research Centre, Fatemieh Hospital, Hamadan, Iran
}

Correspondence should be addressed to I Khodadadi; Email: ikhodadadi@yahoo.com or khodadadi@umsha.ac.ir

\begin{abstract}
It is believed that matrix metalloproteinases (MMPs) play important roles in follicular development and pathogenesis of polycystic ovary syndrome (PCOS). However, conflicting results are available about the alteration of MMP2 and MMP9 concentrations or activities in PCOS. In fact, there is no study entirely investigating both concentration and activity of these MMPs and serum levels of their tissue inhibitors TIMP2 and TIMP1, as well as lipocalin-bound form of MMP9 (MMP9/NGAL). Therefore, the thoroughness of previous studies is questionable. This study was conducted to determine circulatory concentration of MMP2, MMP9, MMP9/NGAL complex, TIMP1 and TIMP2 as well as gelatinase activities of MMP2, MMP9 and MMP9/NGAL complex in women with PCOS and controls. Mean age and BMI as well as serum levels of total cholesterol, triacylglycerol, HDL-C, LDL-C, fasting blood sugar (FBS), insulin, estradiol and sex hormone-binding globulin did not differ between groups, whereas a marked decrease in FSH and significant increases in LH, LH/FSH ratio, testosterone and free androgen index were observed. Women with PCOS and controls showed closed concentrations of MMP2, MMP9, MMP9/NGAL, TIMP1 and TIMP2. Gelatinase activity of MMP9 was found significantly higher in PCOS than in controls $(64.53 \pm 15.32$ vs $44.61 \pm 18.95$ respectively) while patients and healthy subjects showed similar activities of MMP2 and MMP9/NGAL complex. Additionally, PCOS patients showed a higher MMP9/TIMP1 ratio compared with control women. Direct correlations were also observed between circulatory MMP9 level and the concentration and activity of MMP9/NGAL complex. In conclusion, based on the results of present study, we believe that MMP9 may be involved in the pathogenesis of PCOS.
\end{abstract}

Reproduction (2016) 151 305-311

\section{Introduction}

Polycystic ovary syndrome (PCOS) is a prevalent heterogeneous endocrine disorder in women of reproductive age. The worldwide prevalence of $6.5-8 \%$ has been estimated for PCOS based on biochemical and/or clinical evidences (Lujan et al. 2008). PCOS implies the presence of multiple cystic follicles together with hyperandrogenism, chronic oligo or anovulation, hyperinsulinemia, hirsutism and acne (Mason et al. 2008). Remodeling of intra-follicular microenvironment is required for follicle development which is an ordered process coordinately established by various enzymes, cytokines, steroid hormones and growth factors (Dumesic \& Abbott 2008). The preovulatory follicle is enclosed in the extracellular matrix (ECM) that consists of collagen and other ECM components and regulates cellular shape and communication (Dumesic et al. 2008). Rearrangement of this environment requires a coordinated action of proteases and protease inhibitors. Recently, studies have shown that matrix metalloproteinases (MMPs) which play important roles in ECM degradation and re-composition may be implicated in the pathogenesis of PCOS through their effects on ovarian tissue remodeling (Liu et al. 2007). The role of MMPs in ovarian tissue remodeling has recently been demonstrated, and increased circulating levels of MMP2 and MMP9 have been reported in women with PCOS (Lewandowski et al. 2006, Liu et al. 2007, 2008) indicating the involvement of the imbalanced activities of MMPs in PCOS (Shalev et al. 2001).

MMPs are found in all kingdoms of life and comprise a large family of zinc-dependent endoproteinases sharing homologous protein sequences. MMPs can be categorized into six subgroups such as collagenases, gelatinases, stromelysins, matrilysins, membrane-type MMPs and others (Amalinei et al. 2010). MMP2 (gelatinase A, EC 3.4.24.24) and MMP9 (gelatinase B, EC 3.4.24.35) are constitutively expressed by many cell types and seem to have important roles in degradation of a broad spectrum of ECM molecules (Fanjul-Fernandez et al. 2010). It is believed that the observed gelatinolytic activity around the developing follicles and at the apex 
of preovulatory follicles corresponds to the MMP2 and MMP9 (Goldman \& Shalev 2004). The activity of MMPs is regulated at different levels by interactions with endogenous inhibitors such as $\alpha_{2}$-macroglobulin and tissue inhibitors of metalloproteinases (TIMPs) (Nagase et al. 2006), or by proteolytic cleavage of their propeptide domain from the zymogen structures yielding the active enzymes. Four different TIMPs have been identified so far such as TIMP1, TIMP2, TIMP3 and TIMP4. Tissue inhibitors of TIMP1 and TIMP2 are natural inhibitors of MMP9 and MMP2 respectively (Murphy 2011). Therefore, orchestration of the growth and remodeling of cells to their extracellular environment is mediated by a balance between MMPs activities and concentrations of their endogenous inhibitors. In addition, Neutrophil gelatinase-associated lipocalin (NGAL), also known as lipocalin 2, is found to be bound to and positively modulates the activity of MMP9 (Bolignano et al. 2010, Bouchet \& Bauvois 2014). NGAL is an acute phase $25 \mathrm{kDa}$ secretory glycoprotein covalently bounds to MMP9 to form MMP9/NGAL complex which triggers an enhancement of the enzymatic activity of MMP9 through protecting the MMP9 from proteolytic degradation (Bolignano et al. 2010).

A review of the literature showed conflicting results on circulatory concentrations of MMP2 and MMP9. While increased levels of MMP2 and/or MMP9 have shown in PCOS patients (Lewandowski et al. 2006, Liu et al. 2007, 2008), no significant differences have been observed between PCOS and control in the study by Gomes et al. (2011), or a reduced MMP9 level has been reported by Diamanti-Kandarakis et al. (2008). Similar conflicting results are also available about serum TIMP1 and TIMP2 levels (Lewandowski et al. 2006, Liu et al. 2008, Gomes et al. 2011). In addition, a review of the literature showed that not only is there no consistency in the serum levels of MMP2 and MMP9 but also there is no record of serum MMP9 gelatinase activity. Moreover, the only report on MMP2 gelatinase activity showed no difference in MMP2 activity between PCOS patients and healthy subjects (Gomes et al. 2011). Likewise, there is no report on the enzymatic activity of MMP9/NGAL complex in the literature. In fact, simultaneous determination of circulatory concentrations of MMP2, MMP9, TIMP1, TIMP2 and MMP9/NGAL complex together with assessing the gelatinase activities of MMP2, MMP9 and MMP9/NGAL has not been previously carried out in women with PCOS.

In view of all the above and based on the hypothesis that as tissue remodeling in PCOS is more intense than in normal ovaries and some of these parameters may differ between two groups, this study was designed to detect any possible alteration in serum levels of MMP2, MMP9, TIMP1, TIMP2 and MMP9/NGAL complex and any imbalance in the enzyme activities of serum MMP2, MMP9 and MMP9/NGAL complex in women with PCOS compared with healthy individuals.

\section{Materials and methods}

\section{Subjects}

In total, 40 patients with PCOS and 40 healthy, regularly menstruating, women matched for age and BMI, were enrolled in this study. PCOS patients were recruited from the Endometrium and Endometriosis Research Centre of Fatemieh Hospital, Hamadan, Iran. The diagnosis of PCOS was made if there was a history of menstrual irregularity in association with either hyperandrogenism or polycystic ovary morphology, according to the Rotterdam consensus criteria (Rotterdam ESHRE/ASRMSponsored PCOS Consensus Workshop Group 2004). Control women had regular menstrual cycles, normal findings on pelvic ultrasound scan and no features of hyperandrogenism. Exclusion criteria for the study were set as age over 35 years, having cardiovascular disease, Cushing's syndrome, thyroid disease, diabetes mellitus or hypertension, currently being a cigarette smoker and taking medications such as oral contraceptives or metformin that could alter the endocrine parameters. Written informed consent for participation was obtained, and the project was approved by the Research Ethics Committee of Hamadan University of Medical Sciences (Iran).

\section{Samples collection and biochemical assays}

Fasting blood samples of control and PCOS subjects were collected between 0800 and $1000 \mathrm{~h}$ during the early follicular phase (day 2-4 from the day 1 of spontaneous bleeding episode) or any time in an anovulatory phase. Blood samples were centrifuged immediately after collection and aliquots of serum samples were stored at $-70{ }^{\circ} \mathrm{C}$ until analyzed. BMI was calculated as body weight in kilograms divided by height in meters squared $\left(\mathrm{kg} / \mathrm{m}^{2}\right)$.

\section{Biochemical analysis}

The circulating levels of testosterone, luteinizing hormone ( $\mathrm{LH})$, follicle-stimulating hormone $(\mathrm{FSH})$, estradiol and insulin were determined by commercially available sandwich ELISA Kits (Monobind, Inc., Lake Forest, CA, USA) while serum concentration of sex hormone-binding globulin (SHBG) was measured by solid phase IBL ELISA Kit (IBL International GmbH, Humburg, Germany). Free androgen index (FAI) was calculated according to $\mathrm{FAI}$ equation $(\mathrm{FAI}=$ Testosterone $(\mathrm{nmol} / \mathrm{l}) \times 100 / \mathrm{SHBG})$, as previously described (Rosner et al. 2007), and insulin resistance was determined according to the homeostasis model assessment insulin resistance (HOMA-IR) index, using the equation (HOMA$\mathrm{IR}=$ fasting serum glucose $(\mathrm{mM} / \mathrm{l}) \times$ fasting insulin $(\mathrm{mU} / \mathrm{ml}) / 22.5)$ (Matthews et al. 1985). Colorimetric methods were performed to measure glucose, total protein, triacylglycerol (TAG), total cholesterol (TC) and HDL-C using Pars Azmoon Kits (Pars Azmoon Co., Tehran, Iran), and Friedewald equation $(\mathrm{LDL}=\mathrm{TC}-(\mathrm{HDL}+\mathrm{TAG} / 5))$ was used to calculate LDL-C levels (Friedewald et al. 1972).

\section{Determination of serum MMP2, MMP9, MMP9/NGAL, TIMP1 and TIMP2 concentration}

Blood samples were centrifuged immediately after collection and aliquots of serum samples were stored at $-70^{\circ} \mathrm{C}$ until 
analysis. Serum concentration of MMP2, MMP9, TIMP1, TIMP2 and MMP9/NGAL complex was determined using commercially available solid phase ELISA Kits (R\&D Systems, Minneapolis, MN, USA), according to the manufacturer's instruction. The intra- and inter-assay coefficient of variation values were $<5.0$ and $7.5 \%$ respectively, and all measurements were performed in duplicate.

\section{Substrate gel electrophoresis (zymography) for the determination of MMP2, MMP9 and MMP9/NGAL activities}

Gelatinolytic activities of MMP2, MMP9 and MMP9/NGAL complex in serum samples were assessed by zymography, as previously described with some modifications (Lahav-Baratz et al. 2003, Goncalves et al. 2009, Tsai et al. 2011). Briefly, samples were subjected to electrophoresis on a $10 \%$ polyacrylamide-SDS gel (PAGE) co-polymerized with $1 \%$ gelatin as a substrate. The gels underwent electrophoresis until the marker dye reached the bottom of the gel. After electrophoresis, gels were washed twice by gentle agitation (30 min/wash) in $2.5 \%$ Triton $\mathrm{X}-100$ solution at room temperature to enable enzyme renaturation. After decanting the washing solution, gels were immersed in Tris- $\mathrm{HCl}$ buffer, $\mathrm{pH} 7.4$ for $30 \mathrm{~min}$ at room temperature and were then placed in fresh developing buffer for $16 \mathrm{~h}$ at $37^{\circ} \mathrm{C}$. The gels were washed with deionized water and stained with Coomassie Brilliant Blue R-250 (Merck KGaA) in 45\% methanol and 10\% acetic acid for $35 \mathrm{~min}$ at room temperature. The gels were then washed with water and soaked in destaining solution for 3 days until clear bands were seen indicating the MMPs activity of gelatin degradation. Gelatinolytic activities were detected as unstained bands against the background of Coomassie bluestained gelatin. To confirm gelatinase activities observed in unstained bands (corresponding to MMP2, MMP9, and MMP9/ NGAL), experiment was repeated by the immersing of the gels in EDTA buffer which chelates $\mathrm{Zn}^{2+}$ on the active site of MMPs and inhibits enzyme activity leaving no unstained area on the gel (data not shown). Gelatinase activities of MMP2, MMP9 and MMP9/NGAL were reconfirmed by comparing the unstained bands to the standard for MMP2 and MMP9 (R\&D Systems, Inc.). The gels were scanned by Canon LiDE110 scanner (Canon, Inc., Tokyo, Japan), and images were analyzed using TotalLab TL120 software (Nonlinear Dynamics Ltd, Newcastle, UK) to quantify proteolytic activities of MMP2, MMP9 and MMP9/NGAL complex. MMP2 was detected at $60 \mathrm{kDa}$ and reconfirmed by comparing to the standard for MMP2 (R\&D Systems, Inc.). The pro-MMP9 and dimmer forms of MMP9 were identified as bands at 92 and $220 \mathrm{kDa}$ respectively, whereas MMP9/NGAL complex was detected at $135 \mathrm{kDa}$ in comparison with the relative mobility of standard for MMP9 (R\&D Systems, Inc.).

\section{Statistical analysis}

Data were analyzed by SPSS 16 (SPSS, Inc.), and one-sample Kolmogorov-Smirnov test was applied to determine normal distribution of data. Two-tailed unpaired $t$-test used to compare mean differences. One-way ANOVA was used to analyze differences between groups, and linear regression analysis was performed to determine correlation between variables. Results were presented as mean \pm s.D. and the $P$ value $<0.05$ was considered as significant.

\section{Results}

In total, 40 patients with PCOS and 40 healthy, regularly menstruating, women were enrolled in this study. Serum samples were analyzed, and women with PCOS were compared with normal subjects. Table 1 summarizes the clinical, endocrine and biochemical characteristics of the PCOS and healthy women. Patient and healthy subjects were well-matched for age and BMI with no statistically significant differences in age and BMI. Fasting blood sugar (FBS) and serum TAG, TC, HDL-C, LDL-C and SHBG did not differ between groups. Serum insulin levels were slightly higher in women with PCOS compared with controls $(12.19 \pm 9.83$ vs $9.82 \pm$ $4.52 \mu \mathrm{U} / \mathrm{ml}$ respectively), but this difference did not reach statistical significance. Similarly, no difference was observed in HOMA-IR index levels between groups. A marked increase was observed in serum testosterone and FAI in women with PCOS compared with control subjects (Table 1) while the higher estradiol level was not statistically significant. A noticeable decline in $\mathrm{FSH}$ and an increase in $\mathrm{LH}$ levels together with a significant increase in $\mathrm{LH} / \mathrm{FSH}$ ratio were found in PCOS patients.

The results of the present study showed that there is no significant difference in serum concentration of MMP2 or MMP9 between women with PCOS and control (Table 2). Likewise, we showed that serum levels of TIMP1 and TIMP2 did not differ between two groups, although the TIMP2 level did approach significance. Additionally, although a trend to the reduced circulatory MMP9/NGAL levels was observed in PCOS compared with control $(49.90 \pm 6.10$ vs $63.00 \pm 11.02 \mathrm{pg} / \mathrm{ml}$ respectively), statistical analysis showed no significant difference between two groups (Table 2).

Table 1 Clinical, endocrine and biochemical characteristics of women with PCOS and controls.

\begin{tabular}{lccl}
\hline & PCOS $(n=40)$ & Control $(n=40)$ & P value \\
\hline Age $($ year) & $29.52 \pm 3.10$ & $29.70 \pm 5.40$ & 0.820 \\
BMl $\left(\mathrm{kg} / \mathrm{m}^{2}\right)$ & $27.52 \pm 5.03$ & $27.81 \pm 5.36$ & 0.806 \\
FBS $(\mathrm{mg} / \mathrm{dl})$ & $92.97 \pm 19.25$ & $90.62 \pm 16.58$ & 0.560 \\
Triacylglycerol $(\mathrm{mg} / \mathrm{dl})$ & $141.18 \pm 9.07$ & $144.15 \pm 11.53$ & 0.840 \\
Total cholesterol $(\mathrm{mg} / \mathrm{dl})$ & $173.78 \pm 5.54$ & $173.45 \pm 5.55$ & 0.967 \\
HDL-C $(\mathrm{mg} / \mathrm{dl})$ & $43.50 \pm 2.40$ & $41.00 \pm 1.81$ & 0.408 \\
LDL-C $(\mathrm{mg} / \mathrm{dl})$ & $102.04 \pm 4.43$ & $103.62 \pm 5.04$ & 0.814 \\
Insulin $(\mu / \mathrm{l} / \mathrm{ml})$ & $12.19 \pm 9.83$ & $9.82 \pm 4.52$ & 0.173 \\
$\mathrm{HOMA-IR}$ & $2.86 \pm 2.48$ & $2.30 \pm 1.31$ & 0.200 \\
FSH $(\mathrm{mU} / \mathrm{ml})$ & $5.12 \pm 0.34$ & $11.02 \pm 1.33$ & 0.001 \\
LH $(\mathrm{mU} / \mathrm{ml})$ & $9.09 \pm 0.57$ & $5.46 \pm 0.74$ & 0.0002 \\
LH/FSH ratio & $1.91 \pm 0.59$ & $0.87 \pm 0.51$ & 0.001 \\
Estradiol $(\mathrm{pg} / \mathrm{ml})$ & $123.52 \pm 6.50$ & $111.70 \pm 7.64$ & 0.242 \\
Testosterone $(\mathrm{ng} / \mathrm{dl})$ & $3.32 \pm 1.21$ & $0.83 \pm 0.09$ & 0.044 \\
FAl & $27.63 \pm 8.51$ & $7.43 \pm 1.24$ & 0.024 \\
SHBG $(\mathrm{nmol} / \mathrm{l})$ & $55.56 \pm 5.06$ & $60.49 \pm 5.45$ & 0.500 \\
\hline
\end{tabular}


Table 2 Concentration and enzyme activities of MMPs, their tissue inhibitors and MMP9/NGAL in women with PCOS and controls.

\begin{tabular}{lccc}
\hline & PCOS $(n=40)$ & Control $(n=40)$ & P value \\
\hline MMP2 $(\mathrm{pg} / \mathrm{ml})$ & $182.68 \pm 36.76$ & $186.90 \pm 42.44$ & 0.636 \\
MMP9 $(\mathrm{pg} / \mathrm{ml})$ & $436.25 \pm 167.51$ & $441.67 \pm 220.88$ & 0.901 \\
MMP9/NGAL (pg/ml) & $49.90 \pm 38.58$ & $63.00 \pm 69.72$ & 0.303 \\
TIMP1 (pg/ml) & $187.52 \pm 142.47$ & $169.42 \pm 51.16$ & 0.451 \\
TIMP2 (pg/ml) & $41.98 \pm 8.08$ & $39.08 \pm 5.07$ & 0.059 \\
MMP2 activity (AU) & $23.99 \pm 13.26$ & $20.18 \pm 11.14$ & 0.171 \\
MMP9 activity (AU) & $64.53 \pm 15.32$ & $44.61 \pm 18.95$ & 0.002 \\
MMP2act/TIMP2 (AU) & $0.59 \pm 0.36$ & $0.52 \pm 0.28$ & 0.110 \\
MMP9act/TIMP1 (AU) & $0.44 \pm 0.33$ & $0.29 \pm 0.17$ & 0.012 \\
MMP9/NGAL activity & $39.22 \pm 35.96$ & $39.67 \pm 67.72$ & 0.970 \\
$\quad(\mu I U / m l)$ & & & \\
\hline
\end{tabular}

$\mathrm{AU}$ : arbitrary unit.

The results of the present study show that women with PCOS and control subjects had relatively similar levels of serum MMP2 gelatinase activities, whereas a great enhancement $(P=0.002)$ was detected in serum MMP9 enzyme activity in women with PCOS compared with healthy women (Table 2). Moreover, PCOS patients and control women showed a close serum gelatinase activity of MMP9/NGAL complex with no noticeable difference.

Statistical analysis showed a positive correlation between serum concentration and gelatinase activity of MMP2 in control subjects but not in PCOS patients (Table 3), but no correlation was detected between circulatory concentration of MMP9 and its gelatinase activity. The concentration of TIMP1 did not show any correlation with serum level of MMP9 or its gelatinase activity. The circulatory level of TIMP1 neither correlated with MMP9/NGAL concentration nor with its activity (data not shown). In contrast, a significant correlation was observed between circulatory level of TIMP2 and the concentration of MMP2 $(P=0.028)$, but not with MMP2 gelatinase activity (Table 3 ). To assess net MMP2 and MMP9 activities, the MMP2/TIMP2 and MMP9/ TIMP1 ratios were calculated respectively. While PCOS patients and control women did not show different MMP2/ TIMP2 ratios, a significant difference was observed in MMP9/TIMP1 ratio between groups (Table 3).

Serum concentration of MMP9/NGAL complex did not have significant correlation with MMP9/NGAL gelatinase activity, according to the linear regression analysis. Serum concentration of MMP9 showed correlation both with MMP9/NGAL concentration and its gelatinase activity, whereas correlation of MMP9 gelatinase activity was observed neither with MMP9/NGAL complex concentration nor with its activity (Table 3).

\section{Discussion}

PCOS is one of the most common endocrine disorders occurring in women of reproductive age. The underlying etiology of PCOS remains elusive despite being the focus of intense basic and clinical studies (Lujan et al. 2008, Mason et al. 2008). Because of the diverse clinical and metabolic manifestations, considerable debates remain regarding definitive biochemical markers found in PCOS, i.e. although the characteristic abnormalities including hypersecretion of androgens and LH, dyslipidemia, hyperinsulinemia and insulin resistance are known as common laboratory findings in PCOS, these findings have not been consistent in all studies. For instance, while no alteration has been reported in TC, TAG, HDL-C and LDL-C in some studies (DiamantiKandarakis et al. 2008, Cakal et al. 2011, Gomes et al. 2011), a recent report by Sahin et al. (2014) shows significant dyslipidemia in patient with PCOS. Inconsistent findings are also available in the literature showing no alteration (Panidis et al. 2010, Sahin et al. 2014) or increased concentration of insulin in PCOS (Liu et al. 2007, Cakal et al. 2011, Gomes et al. 2011). In contrast to inconsistent reports on blood lipids and insulin, a common surge has been reported in the serum concentration of $\mathrm{LH}$, testosterone and FAI in all conducted studies (Liu et al. 2007, 2008, Panidis et al. 2010, Gomes et al. 2011, Sahin et al. 2014), but the alteration has not been shown in the serum concentration of FSH or HOMA-IR (Liu et al. 2007, 2008, Panidis et al. 2010, Sahin et al. 2014).

The results of the present study showed increased serum levels of $\mathrm{LH}$, testosterone $\mathrm{FAI}$ and $\mathrm{LH} / \mathrm{FSH}$ ratio in line with previous reports (Liu et al. 2007, 2008, Panidis et al. 2010, Gomes et al. 2011, Sahin et al. 2014). We have also showed that circulatory concentrations of TC, TAG, HDL-C, LDL-C, E2, SHBG, insulin and HOMA-IR index did not differ between healthy subjects and women with PCOS. It is plausible that age and BMI are the main players in determining the aforementioned biochemical and hormonal features; therefore, differences in the mean age or BMl of patients (in different studies) may lead to inconsistent results, as seen for insulin resistance which is higher in overweight and obese women than in lean individuals (Livadas et al. 2014, Moran et al. 2015).

Remodeling of intra-follicular microenvironment and follicle development requires a coordinated action of proteases and protease inhibitors. It is believed that

Table 3 Correlations between concentration and enzyme activities of MMPs, their tissue inhibitors and MMP9/NGAL.

\begin{tabular}{llcc}
\hline \multicolumn{2}{c}{ Correlation of variables } & $\boldsymbol{R}^{\mathbf{2}}$ & $\boldsymbol{P}$ value \\
\hline MMP2 conc & TIMP2 conc & 0.245 & 0.028 \\
MMP2 conc & MMP2 act & 0.147 & 0.196 \\
MMP2 act & TIMP2 conc & 0.168 & 0.139 \\
MMP9 conc & TIMP1 conc & 0.092 & 0.418 \\
MMP9 conc & MMP9 act & 0.082 & 0.472 \\
MMP9 conc & MMP9/NGAL conc & 0.274 & 0.014 \\
MMP9 conc & MMP9/NGAL act & 0.224 & 0.048 \\
MMP9 act & TIMP1 conc & 0.008 & 0.944 \\
MMP9 act & MMP9/NGAL conc & 0.172 & 0.128 \\
MMP9 act & MMP9/NGAL act & 0.022 & 0.189 \\
MMP9/NGAL conc & MMP9/NGAL act & 0.092 & 0.421 \\
\hline
\end{tabular}

act: activity; conc: concentration. 
MMPs may be implicated in the pathogenesis of PCOS through their effect on ovarian tissue remodeling (Liu et al. 2007). It is postulated that the orchestration of the growth and remodeling of follicles to their extracellular environment is mediated by a balance between MMPs activities and concentrations of their endogenous inhibitors (TIMPs). However, conflicting results have been reported during the last few years about the involvement of MMPs and/or their tissue inhibitors in the pathogenesis of PCOS. There is no extensive body of literature investigating the role of MMP2 in women with PCOS, and the circulatory concentration of MMP2 has only been investigated in two studies indicating an increased level of MMP2 in PCOS (Lewandowski et al. 2006, Liu et al. 2007). Intriguingly, here we showed a close concentration of MMP2 in healthy women and women with PCOS. The observed disagreement between our results and the results of previous studies might be due to the low number of subjects recruited in previous works by Lewandowski et al. (2006) and Liu et al. (2007) (35 and 45 subjects respectively) compared with 80 subjects enrolled in the present study.

Although an increased serum level of MMP9 has been reported in some studies (Lewandowski et al. 2006, Liu et al. 2008), there are also evidences in literature indicating a marked decline (Diamanti-Kandarakis et al. 2008) or even no alteration in the plasma MMP9 concentration in PCOS patients (Gomes et al. 2011). These conflicting results may indicate that in comparison to the enzymatic activities, the concentration of MMPs may not play a pivotal role in the pathogenesis of PCOS. Concordant with this explanation, we showed that there is no difference in MMP9 concentration between healthy subjects and women with PCOS. We also showed that the concentrations of TIMP1 and TIMP2 did not differ between groups.

The balance between MMPs and their tissue inhibitors is many-sided. For instance, although TIMP1 and TIMP2 inhibit MMP9 and MMP2 respectively, the pro-enzyme (zymogen) forms of these MMPs may also bind to and inhibited by TIMP3 in the body (Murphy 2011). Therefore, consistent correlations have not been observed between the concentration of MMP2 or MMP9 and the serum levels of their corresponding tissue inhibitors in previous works. Accordingly, it has shown that there is no synchronized changes between serum MMP9 and TIMP1 (Lewandowski et al. 2006, Liu et al. 2008, Gomes et al. 2011), or the concentration of TIMP2 is not following the alteration in serum level of MMP2 (Lewandowski et al. 2006). Likewise, we showed that the concentration of TIMP1 is not correlated to serum MMP9 level. This observation is in line with the results reported by Gomes et al. (2011) confirming neither changes in the concentrations of MMP9 and TIMP1 nor existence of any correlation between these concentrations in PCOS. Unlike MMP9, in the present study, MMP2 has evinced a significant correlation with its corresponding tissue inhibitor (TIMP2). A similar correlation between the concentrations of MMP2 and TIMP2 has recently been reported in follicular fluid (Baka et al. 2010) but not in the serum of women with PCOS (Lewandowski et al. 2006). However, there is no compelling evidences in the literature to show concomitant change of MMPs concentrations/activities in serum and follicular fluid. It is plausible that in PCOS, the concentration of MMPs and TIMPs is more prone to be affected in follicular fluid than in circulation. To assess net MMP2 and MMP9 activities, the MMP2/ TIMP2 and MMP9/TIMP1 ratios were calculated respectively. The MMP2/TIMP2 ratio was slightly higher in women with PCOS compared with controls, but this difference did not reach statistical significance, whereas a marked difference was observed in MMP9/TIMP1 ratio between groups (Table 3). A similar increased MMP9/ TIMP1 ratio has previously been reported in serum (Liu et al. 2008) and follicular fluid (Gomes et al. 2011) samples in PCOS patients. Together these observations confirm alteration of MMP9 activity in PCOS patients and, therefore, provide its potential role in the pathogenesis of the disease.

Considering the literature data, the present study is the first study that simultaneously determines circulatory concentrations of MMP2, MMP9, TIMP1, TIMP2 and MMP9/NGAL complex together with the determination of gelatinase activities of MMP2, MMP9 and MMP9/NGAL in women with PCOS. We showed that the serum concentration and activity of MMP9/NGAL did not differ between groups. Although the decreased concentration of MMP9/NGAL complex has recently been reported in PCOS (Diamanti-Kandarakis et al. 2008), there is no information available in the literature about the gelatinase activity of MMP9/NGAL complex. Supposedly, the disagreement between our findings and previous report by Diamanti-Kandarakis et al. (2008) can be explained by the fact that the concentration of NGAL, which binds to MMP9 and protects the MMP9 from proteolytic degradation (Bolignano et al. 2010, Bouchet \& Bauvois 2014), is closely correlated with insulin, insulin resistance and testosterone (Cakal et al. 2011). Therefore, differences in hormonal features of subjects in different studies may affect the concentration and/or activity of MMP9/NGAL complex.

Here for the first time, we also showed that women with PCOS exhibit much higher gelatinase activity of MMP9 than control subjects, whereas there was no difference in the activity of MMP2 between groups. Our later observation is supported by the previous reports indicating that the activity of MMP2 in serum (Gomes et al. 2011) or follicular fluid (Lahav-Baratz et al. 2003) does not alter in PCOS. However, there is no such a record in the literature about MMP9, since the activity of MMP9 has not been previously investigated in PCOS. Therefore, regarding the higher MMP9 activity in PCOS, we have strengthened the hypothesis of involvement of 
MMP9 gelatinase activity in the pathogenesis of PCOS. This finding is of immense importance, since it is the first report on the association of PCOS with MMP9 activity. The importance of contribution of MMP9 in PCOS, as observed here, is boosted by augmenting results showing significant correlation of serum MMP9 level with either MMP9/NGAL concentration or activity.

There are, however, arguments in literature on using serum samples to assess MMP9 concentration or activity. Some authors have argued that blood coagulation process results the release of MMPs by platelets or leukocytes during platelet activation and, thus, leads to artificially higher MMP9 levels in serum compared to citrate and heparin plasma samples (Gerlach et al. 2005, 2007, Souza-Tarla et al. 2005). This notion was supported by further observations indicating that serum MMP9 levels did not correlate with the levels found in plasma samples (Gerlach et al. 2007). However, we still believed that serum MMP9 levels, as determined in the present study, may reasonably be valid, since first, MMP9 levels have been determined in serum samples of both patients and control women; therefore, any methodological pitfalls or artifact increment in MMP9 levels may occur evenly in all samples. Secondly, very recent study has revealed the existence of good correlation between serum and plasma concentrations of MMP9 when evaluating alterations in the concentration of this marker in disease conditions (Gerlach et al. 2009). Therefore, serum samples could simply be used instead of plasma samples when comparing concentrations in a particular study (Gerlach et al. 2009).

Taking in to account the limitations present in our study, we believe that the usage of citrate or heparin plasma samples instead of serum samples, determination of circulatory lipocalin-2 (NGAL) in its free unbound form, assessment of active forms of MMPs rather than total MMPs (pro-MMPs plus active MMPs) and measurement of MMP2, MMP9, TIMP1 and TIMP2 gene expression levels may strengthen the results. Considering the literature data, as i) age and BMI which are two important players in determining hormonal and biochemical features, differed in previous studies, ii) the low number of subjects recruited in previous studies and iii) the outcomes of previous studies are conflicting, conducting further investigations which entirely overcome all confounder factors by the enrolment of larger number of subjects, pair matching of control and patients in age and BMI and simultaneous determination of gelatinase activity as well as concentration and gene expression levels of MMPs is required. In addition, since most of the clinical studies have determined only the total MMP2 and/or MMP9 and it is not clear whether the total MMP forms or the activated forms are of diagnostic value for diseases, separate analysis of these markers is needed.

In conclusion, our results have shown that PCOS patients exhibit higher MMP9 gelatinase activity and
MMP9/TIMP1 ratio than healthy control women. In addition, direct associations have been observed between the serum concentration of MMP9 with either MMP9/NGAL concentration or its activity. Therefore, MMP9 may involve in the pathogenesis of PCOS.

\section{Declaration of interest}

The authors declare that there is no conflict of interest that could be perceived as prejudicing the impartiality of the research reported.

\section{Funding}

This research was supported by Hamadan University of Medical Sciences, as MSc thesis, and did not receive any specific grant from any funding agency in the public, commercial or not-for-profit sector.

\section{Acknowledgements}

We would like to thank the staffs of the laboratory of Endometrium and Endometriosis Research Centre of Fatemieh Hospital for their assistance in enrolling subjects and conducting the study. We are also very grateful to Dr Poorolajal for his valuable assistance in statistical analysis.

\section{References}

Amalinei C, Caruntu ID, Giusca SE \& Balan RA 2010 Matrix metalloproteinases involvement in pathologic conditions. Romanian Journal of Morphology and Embryology 51 215-228.

Baka S, Zourla K, Kouskouni E, Makrakis E, Demeridou S, Tzanakaki D, Hassiakos D \& Creatsas G 2010 Matrix metalloproteinases 2 and 9 and their tissue inhibitors in the follicular fluid of patients with polycystic ovaries undergoing in vitro fertilisation. In Vivo 24 293-296.

Bolignano D, Donato V, Lacquaniti A, Fazio MR, Bono C, Coppolino G \& Buemi M 2010 Neutrophil gelatinase-associated lipocalin (NGAL) in human neoplasias: a new protein enters the scene. Cancer Letters $\mathbf{2 8 8}$ 10-16. (doi:10.1016/j.canlet.2009.05.027)

Bouchet S \& Bauvois B 2014 Neutrophil gelatinase-associated lipocalin (NGAL), pro-matrix metalloproteinase-9 (pro-MMP-9) and their complex pro-MMP-9/NGAL in leukaemias. Cancers 6 796-812. (doi:10.3390/ cancers6020796)

Cakal E, Ozkaya M, Engin-Ustun Y \& Ustun Y 2011 Serum lipocalin-2 as an insulin resistance marker in patients with polycystic ovary syndrome. Journal of Endocrinological Investigation 34 97-100. (doi:10.1007/ BF03347037)

Diamanti-Kandarakis E, Livadas S, Kandarakis SA, Margeli A \& Papassotiriou I 2008 Serum concentrations of atherogenic proteins neutrophil gelatinase-associated lipocalin and its complex with matrix metalloproteinase- 9 are significantly lower in women with polycystic ovary syndrome: hint of a protective mechanism? European Journal of Endocrinology 158 525-531. (doi:10.1530/EJE-07-0822)

Dumesic DA \& Abbott DH 2008 Implications of polycystic ovary syndrome on oocyte development. Seminars in Reproductive Medicine 26 53-61. (doi:10.1055/s-2007-992925)

Dumesic DA, Padmanabhan V \& Abbott DH 2008 Polycystic ovary syndrome and oocyte developmental competence. Obstetrical \& Gynecological Survey 63 39-48. (doi:10.1097/OGX.0b013e31815e85fc)

Fanjul-Fernandez M, Folgueras AR, Cabrera S \& Lopez-Otin C 2010 Matrix metalloproteinases: evolution, gene regulation and functional analysis in mouse models. Biochimca et Biophysica Acta 1803 3-19. (doi:10.1016/ j.bbamcr.2009.07.004) (doi:10.1016/j.bbamcr.2009.07.004) 
Friedewald WT, Levy RI \& Fredrickson DS 1972 Estimation of the concentration of low-density lipoprotein cholesterol in plasma, without use of the preparative ultracentrifuge. Clinical Chemistry 18 499-502.

Gerlach RF, Uzuelli JA, Souza-Tarla CD \& Tanus-Santos JE 2005 Effect of anticoagulants on the determination of plasma matrix metalloproteinase (MMP)-2 and MMP-9 activities. Analytical Biochemistry 344 147-149. (doi:10.1016/j.ab.2005.04.038)

Gerlach RF, Demacq C, Jung K \& Tanus-Santos JE 2007 Rapid separation of serum does not avoid artificially higher matrix metalloproteinase (MMP)9 levels in serum versus plasma. Clinical Biochemistry 40 119-123. (doi:10.1016/j.clinbiochem.2006.10.007)

Gerlach RF, Meschiari CA, Marcaccini AM, Palei AC, Sandrim VC, Cavalli RC \& Tanus-Santos JE 2009 Positive correlations between serum and plasma matrix metalloproteinase (MMP)-2 or MMP-9 levels in disease conditions. Clinical Chemistry and Laboratory Medicine 47 888-891. (doi:10.1515/CCLM.2009.203)

Goldman S \& Shalev E 2004 MMPS and TIMPS in ovarian physiology and pathophysiology. Frontiers in Bioscience 9 2474-2483. (doi:10. 2741/1409)

Gomes VA, Vieira CS, Jacob-Ferreira AL, Belo VA, Soares GM, Fernandes JB, Ferriani RA \& Tanus-Santos JE 2011 Imbalanced circulating matrix metalloproteinases in polycystic ovary syndrome. Molecular and Cellular Biochemistry 353 251-257. (doi:10.1007/ s11010-011-0793-6)

Goncalves FM, Jacob-Ferreira AL, Gomes VA, Casella-Filho A, Chagas AC, Marcaccini AM, Gerlach RF \& Tanus-Santos JE 2009 Increased circulating levels of matrix metalloproteinase (MMP)-8, MMP-9, and pro-inflammatory markers in patients with metabolic syndrome. Clinica Chimica Acta 403 173-177. (doi:10.1016/j.cca.2009.02.013)

Lahav-Baratz S, Kraiem Z, Shiloh H, Koifman M, Ishai D \& Dirnfeld M 2003 Decreased expression of tissue inhibitor of matrix metalloproteinases in follicular fluid from women with polycystic ovaries compared with normally ovulating patients undergoing in vitro fertilization. Fertility and Sterility 79 567-571. (doi:10.1016/S0015-0282(02)04838-0)

Lewandowski KC, Komorowski J, O'Callaghan CJ, Tan BK, Chen J, Prelevic GM \& Randeva HS 2006 Increased circulating levels of matrix metalloproteinase-2 and -9 in women with the polycystic ovary syndrome. Journal of Clinical Endocrinology and Metabolism 91 1173-1177. (doi:10.1210/jc.2005-0648)

Liu B, Guan YM \& Zheng JH 2007 Elevated serum levels of matrix metalloproteinase-2 in women with polycystic ovarian syndrome. International Journal of Gynaecology and Obstetrics 96 204-205. (doi:10.1016/j.ijgo.2006.11.012)

Liu B, Cai LY, Lv HM, Xia L, Zhang YJ, Zhang HX \& Guan YM 2008 Raised serum levels of matrix metalloproteinase-9 in women with polycystic ovary syndrome and its association with insulin-like growth factor binding protein-1. Gynecological Endocrinology 25 285-288. (doi:10.1080/09513590802056995)

Livadas S, Kollias A, Panidis D \& Diamanti-Kandarakis E 2014 Diverse impacts of aging on insulin resistance in lean and obese women with polycystic ovary syndrome: evidence from 1345 women with the syndrome. European Journal of Endocrinology 171 301-309. (doi:10.1530/ EJE-13-1007)

Lujan ME, Chizen DR \& Pierson RA 2008 Diagnostic criteria for polycystic ovary syndrome: pitfalls and controversies. Journal of Obstetrics and Gynaecology Canada 30 671-679.
Mason H, Colao A, Blume-Peytavi U, Rice S, Qureshi A, Pellatt L, Orio F \& Atkin SL 2008 Polycystic ovary syndrome (PCOS) trilogy: a translational and clinical review. Clinical Endocrinology 69 831-844. (doi:10.1111/ j.1365-2265.2008.03329.x)

Matthews DR, Hosker JP, Rudenski AS, Naylor BA, Treacher DF \& Turner RC 1985 Homeostasis model assessment: insulin resistance and $\beta$-cell function from fasting plasma glucose and insulin concentrations in man. Diabetologia 28 412-419. (doi:10.1007/BF00280883)

Moran C, Arriaga M, Arechavaleta-Velasco F \& Moran S 2015 Adrenal androgen excess and body mass index in polycystic ovary syndrome. Journal of Clinical Endocrinology and Metabolism 100 942-950. (doi:10. 1210/jc.2014-2569)

Murphy G 2011 Tissue inhibitors of metalloproteinases. Genome Biology 12 233. (doi:10.1186/gb-2011-12-11-233)

Nagase H, Visse R \& Murphy G 2006 Structure and function of matrix metalloproteinases and TIMPs. Cardiovascular Research 69 562-573. (doi:10.1016/j.cardiores.2005.12.002)

Panidis D, Tziomalos K, Koiou E, Kandaraki EA, Tsourdi E, Delkos D, Kalaitzakis E \& Katsikis I 2010 The effects of obesity and polycystic ovary syndrome on serum lipocalin-2 levels: a cross-sectional study. Reproductive Biology and Endocrinology 8 1-8. (doi:10.1186/14777827-8-151)

Rosner W, Auchus RJ, Azziz R, Sluss PM \& Raff H 2007 Position statement: utility, limitations, and pitfalls in measuring testosterone: an Endocrine Society position statement. Journal of Clinical Endocrinology and Metabolism 92 405-413. (doi:10.1210/jc.2006-1864)

Rotterdam ESHRE/ASRM-Sponsored PCOS Consensus Workshop Group 2004 Revised 2003 consensus on diagnostic criteria and long-term health risks related to polycystic ovary syndrome. Fertility and Sterility $\mathbf{8 1}$ 19-25.

Sahin SB, Cure MC, Ugurlu Y, Ergul E, Gur EU, Alyildiz N \& Bostan M 2014 Epicardial adipose tissue thickness and NGAL levels in women with polycystic ovary syndrome. Journal of Ovarian Research 7 1-6. (doi:10. 1186/1757-2215-7-24)

Shalev E, Goldman S \& Ben Shlomo I 2001 The balance between MMP-9 and MMP-2 and their tissue inhibitor (TIMP)-1 in luteinized granulosa cells: comparison between women with PCOS and normal ovulatory women. Molecular Human Reproduction 7 325-331. (doi:10.1093/ molehr/7.4.325)

Souza-Tarla CD, Uzuelli JA, Machado AA, Gerlach RF \& Tanus-Santos JE 2005 Methodological issues affecting the determination of plasma matrix metalloproteinase (MMP)-2 and MMP-9 activities. Clinical Biochemistry 38 410-414. (doi:10.1016/j.clinbiochem.2005.02.010)

Tsai HC, Ye SY, Kunin CM, Lee SS, Wann SR, Tai MH, Shi MH, Liu YC \& Chen YS 2011 Expression of matrix metalloproteinases and their tissue inhibitors in the serum and cerebrospinal fluid of patients with HIV-1 infection and syphilis or neurosyphilis. Cytokine 54 109-116. (doi:10.1016/j.cyto.2011.02.007)

Received 25 July 2015

First decision 8 September 2015

Revised manuscript received 22 December 2015

Accepted 5 January 2016 